\title{
Corrélats du bien-être chez les Canadiens présentant des troubles de l'humeur ou d'anxiété
}

\author{
H. Orpana, Ph. D. (1,2); J. Vachon, M. Sc. (1); C. Pearson, M.A. (1); K. Elliott, Ph. D. (2); M. Smith, M. Sc. (3); B. Branchard (1)
}

Cet article a fait l'objet d'une évaluation par les pairs.

Diffuser cet article sur Twitter

\section{Résumé}

Introduction : Notre objectif visait à étudier les variables liées au bien-être, mesurées par une autoévaluation de la santé mentale (AESM) très positive et une satisfaction élevée à l'égard de la vie (SV) chez des adultes canadiens (âgés de 18 ans et plus) présentant un trouble de l'humeur ou d'anxiété.

Méthodologie : Nous avons utilisé des données nationales représentatives tirées de l'Enquête sur les personnes ayant une maladie chronique au Canada - Composante des troubles de l'humeur et d'anxiété (EPMCC-TAH) de 2014 afin de décrire l'association entre bien-être et comportements d'autogestion (activité physique, sommeil et méditation) ainsi que stress, adaptation et soutien social perçus. Nous avons eu recours à une régression logistique multivariée pour modéliser la relation entre ces facteurs et les mesures du bien-être.

Résultats : Environ une personne sur trois atteinte d'un trouble de l'humeur ou d'anxiété a fait état d'une AESM positive. Les modèles de régression logistique ont révélé que plusieurs caractéristiques, comme un âge plus avancé, une autoévaluation de la santé générale positive, des limitations fonctionnelles moins nombreuses ainsi que la perception d'un moindre stress à l'égard de la vie, de meilleures capacités d'adaptation et d'un plus grand soutien social, étaient associées à des niveaux de bien-être plus élevés. Les comportements d'autogestion (entamer une activité physique, méditer, adopter de saines habitudes de sommeil et atteindre un certain nombre d'heures de sommeil chaque nuit) n'étaient pas significativement associés à des mesures du bien-être dans notre modèle multivarié.

Conclusion : Les adultes canadiens souffrant de troubles de l'humeur ou d'anxiété qui ont déclaré percevoir un niveau de stress plus faible, un plus grand soutien social et une meilleure capacité d'adaptation étaient plus susceptibles de déclarer également des scores de bien-être plus élevés. Cette étude a fourni des données probantes à partir d'un échantillon représentatif de la population montrant qu'il est possible de vivre dans un état de bien-être même en présence d'un trouble de l'humeur ou d'anxiété.

Mots-clés : troubles de l'humeur, troubles d'anxiété, bien-être, autoévaluation de la santé mentale, satisfaction à l'égard de la vie, adultes, Canada

\section{Introduction}

La Stratégie en matière de santé mentale pour le Canada, Changer les orientations, changer des vies, encourage les personnes atteintes d'un trouble mental à changer leur perception à l'égard de leur maladie et de leurs symptômes en se concentrant davantage sur le concept de rétablissement, c'est-à-dire sur la « possibilité de mener une vie satisfaisante, pleine d'espoir et utile » en présence d'un trouble de santé mentale $^{1}$. Le bien-être est un état positif défini le plus souvent en termes de bienêtre hédonique ou émotionnel et de bienêtre eudémonique ou fonctionnel ${ }^{2}$. Le

\section{Points saillants}

- Un Canadien sur trois atteint d'un trouble de l'humeur ou d'anxiété a autoévalué sa santé mentale comme très bonne ou excellente. L'échantillon a fait état d'un taux relativement élevé de satisfaction à l'égard de la vie. Le niveau de bien-être était à son plus bas chez les personnes atteintes à la fois d'un trouble de l'humeur et d'un trouble d'anxiété.

- Les degrés de perception du stress, de la capacité d'adaptation et du soutien social étaient significativement associés au bien-être. Nous n'avons toutefois pas observé d'associations significatives entre les comportements d'autogestion et le bien-être.

- Il est possible de souffrir d'un trouble de l'humeur ou d'anxiété et de jouir d'un grand bien-être.

bien-être hédonique implique souvent la présence d'une satisfaction à l'égard de la vie et d'émotions positives, alors que le bien-être eudémonique implique plutôt des notions comme la réalisation de soi et la découverte d'un sens à sa vie.

L’Agence de la santé publique du Canada (ASPC) comme l'Organisation mondiale de la santé (OMS) abordent la question de la santé mentale sous l'angle du bien-être. L'ASPC définit la santé mentale positive comme étant " la capacité qu'a chacun d'entre nous de ressentir, de penser et d'agir de manière à améliorer notre aptitude à jouir de la vie et à relever les défis auxquels nous sommes confrontés. Il s'agit

Rattachement des auteurs :

1. Agence de la santé publique du Canada, Ottawa (Ontario), Canada

2. École de psychologie, Université d'Ottawa, Ottawa (Ontario), Canada

3. Centre manitobain des politiques en matière de santé, College of Medicine, Faculty of Health Sciences, Université du Manitoba, Winnipeg (Manitoba), Canada

Correspondance : Heather Orpana, Division de l'intégration scientifique, Direction des déterminants sociaux et de l'intégration scientifique, Direction générale de la promotion de la

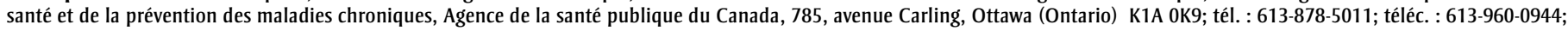
courriel : heather.orpana@phac-aspc.gc.ca 
d'un sentiment positif de bien-être émotionnel et spirituel qui respecte l'importance de la culture, de l'équité, de la justice sociale, des interactions et de la dignité personnelle $»^{3}$. Cette définition est similaire à celle de l'Organisation mondiale de la santé, pour laquelle la santé mentale est : " un état de bien-être qui permet à chacun de réaliser son potentiel, de faire face aux difficultés normales de la vie, de travailler avec succès et de manière productive et d'être en mesure d'apporter une contribution à la communauté » .

Le concept de qualité de vie (QV) est lié à celui de bien-être mais distinct de ce dernier. L’OMS définit la qualité de vie comme la " perception qu'a un individu de sa place dans l'existence, dans le contexte de la culture et du système de valeurs dans lesquels il vit, en relation avec ses objectifs, ses attentes, ses normes et ses inquiétudes $»^{5}$. Parmi les nombreuses mesures de la qualité de vie, on compte l'évaluation subjective de la satisfaction à l'égard de la vie (SV), dans son ensemble ou relativement à des composantes précises.

D'après des travaux de recherche portant sur les personnes vivant avec un trouble de l'humeur, le bien-être est associé à des niveaux supérieurs de fonctionnement géné$\mathrm{ral}^{6}$, et un certain nombre d'interventions à l'égard du mode de vie se sont avérées prometteuses pour accroitre le bien-être? Cependant, la plupart des études portant sur le bien-être des personnes atteintes d'un trouble de l'humeur ou d'anxiété sont menées auprès d'échantillons cliniques de petite taille et qui ne sont pas représentatifs de la population canadienne. Cette étude vise à examiner les facteurs associés au bien-être, mesurés par une autoévaluation de la santé mentale (AESM) positive et une grande satisfaction à l'égard de la vie (SV) en général au sein d'un échantillon représentatif de la population canadienne présentant un trouble de l'humeur ou d'anxiété, dans le cadre de l'Enquête sur les personnes ayant une maladie chronique au Canada (EPMCC) de 2014. Ces facteurs relèvent de plusieurs comportements d'autogestion ainsi que du stress, de la capacité d'adaptation et du soutien social perçus.

Comportements d'autogestion dans les cas de troubles de l'humeur ou d'anxiété

Plusieurs comportements sont associés à l'atténuation des symptômes et à de meilleurs résultats cliniques chez les personnes atteintes d'un trouble de l'humeur ou d'anxiété. Dans le cadre de notre étude, les " comportements d'autogestion » sont d'entamer une activité physique ou la méditation pour mieux gérer son trouble et d'adopter de saines habitudes de sommeil aussi bien que d'atteindre la quantité de sommeil recommandée, mesurée par le nombre habituel d'heures de sommeil par nuit. Ces comportements peuvent être amorcés par la personne ou suggérés par un professionnel de la santé dans le cadre d'une approche holistique de traitement. Chacun de ces comportements est décrit cidessous dans le contexte du bien-être.

\section{Activité physique}

Les personnes atteintes d'un trouble de l'humeur ou d'anxiété peuvent adopter, de leur plein gré ou sur la recommandation d'un professionnel de la santé dans le cadre d'une approche de soins cliniques, une vaste gamme de comportements favorisant le bien-être. Non seulement les bienfaits de l'activité physique sont prouvés dans la littérature pour l'atténuation des symptômes chez les personnes souffrant d'un trouble de l'humeur ${ }^{8}$, mais de plus en plus de données probantes montrent que l'activité physique est également susceptible d'améliorer le bien-être des personnes souffrant de dépression majeure ou présentant des symptômes de dépression ${ }^{8-10}$. Par exemple, Galper et collab. ${ }^{10}$ ont établi une relation dose-réponse positive croissante entre les capacités cardiorespiratoires et l'état de bien-être général moyen estimé, de même qu'une relation étroite entre le volume d'activité physique et les scores de bien-être général estimés.

Le lien entre les troubles d'anxiété et l'activité physique a été étudié moins souvent, et les réponses à l'exercice semblent varier selon le type de trouble d'anxiétée, ${ }^{8,1}$. Il existe néanmoins des données préliminaires selon lesquelles on peut associer l'activité physique à un état de bien-être accru chez les personnes atteintes de certains types de troubles d'anxiété, comme l'état de stress post-traumatique, le trouble d'anxiété généralisée, le trouble d'anxiété sociale et le trouble obsessionnel-compulsif ${ }^{12}$.

\section{Sommeil}

Le dysfonctionnement du sommeil est associé aux troubles de l'humeur et d'anxiété $^{13}$. Le nombre d'heures de sommeil et la qualité du sommeil chez les personnes atteintes d'un trouble de l'humeur ou d'anxiété sont également liés aux mesures du bien-être. Par exemple, Hamilton et collab. ${ }^{14}$ ont constaté que des répondants souffrant d'un trouble dépressif majeur (TDM) et dormant entre 6 et 8,5 heures par nuit éprouvent un bien-être psychologique supérieur, d'après les six dimensions du bien-être psychologique de l'échelle de Ryff $^{15}$, que ceux qui cumulent plus d'heures ou moins d'heures de sommeil. Par ailleurs, d'après Peth et collab. ${ }^{16}$, les participants ayant reçu un diagnostic de TDM et qui avaient la possibilité de faire une sieste au cours de la journée ont déclaré jouir d'un meilleur bien-être subjectif que ceux qui étaient maintenus éveillés avec des activités contrôlées au cours de la même période.

\section{Méditation}

Le recours à la méditation, plus particulièrement à la méditation « de pleine conscience », dans le traitement des troubles de l'humeur et d'anxiété s'est révélé prometteur dans de nombreuses études portant sur des traitements ${ }^{17}$. D'autres techniques de méditation, comme la méditation fondée sur la bienveillance, se sont aussi révélées prometteuses, même si leurs effets sur le bienêtre des participants atteints d'un trouble de l'humeur étaient variables ${ }^{18}$. Dans le cadre d'une étude qualitative sur les stratégies d'adaptation individuelles, de nombreux participants ayant reçu un diagnostic de trouble bipolaire (un trouble de l'humeur) ont déclaré avoir recours à la méditation pour gérer eux-mêmes les expériences négatives attribuables à leur trouble ${ }^{19}$.

\section{Stress, adaptation et soutien social}

Il existe des relations bien étayées entre le stress, la capacité d'adaptation et le soutien social et le bien-être. Une exposition prolongée à des facteurs de stress peut accrô̂tre le risque de développer des troubles mentaux et des problèmes de santé physique $^{20,21}$, et il existe un rapport inverse entre le niveau de stress perçu et le bienêtre $^{22}$. La capacité d'adaptation se définit par un ensemble de stratégies cognitives et comportementales adoptées par une personne pour faire face aux difficultés découlant de situations stressantes, comme la résolution de problèmes ou la régulation émotionnelle ${ }^{23}$. Certaines interventions visant à accroître les capacités d'adaptation positives ont été associées à des niveaux de bien-être accrus $^{24}$. Le soutien social est invariablement associé au bien-être chez les personnes ayant un trouble de l'humeur ou d'anxiété. Par exemple, Kuehner et 
Buerger $^{25}$ ont constaté que la présence d'un partenaire intime était associée à des scores plus élevés en matière de qualité de vie subjective, dans les domaines psychologique et des relations sociales, chez les participants souffrant de dépression unipolaire. Panayiotou et Karekla ${ }^{26}$ ont constaté que, chez les participants ayant reçu un diagnostic de trouble d'anxiété généralisée, de trouble d'anxiété sociale ou de trouble panique, la perception d'un soutien social accru était un prédicteur de qualité de vie psychologique supérieure.

Bien que les éléments de preuve associant ces facteurs au bien-être des personnes atteintes d'un trouble de l'humeur ou d'anxiété soient de plus en plus nombreux, il est de toute évidence nécessaire de poursuivre les recherches afin de mieux comprendre les corrélats du bien-être au sein de cette population. Jusqu'à présent, la plupart des études se sont concentrées sur ces relations au sein d'échantillons de commodité de petite taille et dans le cadre d'études cliniques de faible envergure. Les travaux de recherche portant sur les personnes atteintes de troubles mentaux sont souvent centrés sur les résultats négatifs (détresse et dysfonctionnement), même si le bien-être est reconnu comme une composante importante et réalisable chez les personnes souffrant de troubles mentaux ${ }^{1}$. Notre étude pourra donc s'ajouter à cette littérature, grâce à l'analyse du bien-être au moyen d'un échantillon représentatif de Canadiens souffrant d'un trouble anxieux ou de l'humeur, ainsi que de l'association entre les comportements d'autogestion, les facteurs de stress, d'adaptation et de soutien social et les résultats à l'autoévaluation de la santé mentale (AESM) et de la satisfaction à l'égard de la vie (SV) au sein de cet échantillon. La documentation accessible au moment de cette étude nous a conduit à faire l'hypothèse d'une association positive entre d'une part chacun des comportements d'autogestion, la réduction du stress et l'amélioration de la capacité d'adaptation et du soutien social et d'autre part des résultats supérieurs à l'AESM et de la SV.

\section{Méthodologie}

Nous avons analysé les données tirées de l'Enquête sur les personnes ayant une maladie chronique au Canada - Composante des troubles de l'humeur et d'anxiété (EPMCC-TAH) de 2014. La population étudiée était constituée de 3361 personnes de 18 ans et plus ayant déclaré, dans le cadre de l'Enquête sur la santé dans les collectivités canadiennes de 2013, avoir reçu un diagnostic de trouble de l'humeur ou d'anxiété établi par un professionnel de la santé. La méthode de collecte de données et les détails sur l'échantillon de l'EPMCC-TAH sont décrits dans le document d'orientation méthodologique joint au présent numéro de la revue Promotion de la santé et prévention des maladies chroniques au Canada ${ }^{27}$.

\section{Bien-être}

Nous avons utilisé deux mesures du bienêtre dans le cadre de cette étude, soit l'AESM et la SV. L'AESM se définit comme l'évaluation que fait une personne de sa santé mentale en général. Nous avons considéré que les personnes ayant évalué leur santé mentale comme étant " excellente » ou « très bonne " avaient un score d'AESM élevé, et que celles ayant déclaré avoir une santé mentale "bonne », " passable » ou " mauvaise » avaient un score d'AESM faible. En dichotomisant la variable de cette façon, on crée un groupe qui cadre avec la manière dont Statistique Canada et l'ASPC rendent compte de cet indicateur ${ }^{28,29}$.

La mesure de la satisfaction à l'égard de la vie est une évaluation ou un jugement global de la satisfaction qu'éprouve une personne à l'égard de sa vie, en fonction de ses propres critères $^{30}$. Conformément aux lignes directrices de l'Organisation de Coopération et de Développement Économiques concernant la mesure du bien-être subjectif, les participants devaient répondre à la question suivante : « À l'aide d'une échelle de 0 à 10 , où 0 signifie "très insatisfait” et 10 signifie "très satisfait”, quel sentiment éprouvez-vous maintenant en général à l'égard de votre vie? ». L'analyse des données sur la SV a révélé que des réponses étaient faussées et qu'elles ne correspondaient pas à une distribution normale. De ce fait, nous n'avons pas utilisé ces réponses comme variable continue pour l'analyse. Nous avons préféré dichotomiser l'échelle au moyen d'une médiane : niveau élevé de SV (scores de 8 et plus) et faible niveau de SV (scores inférieurs à 8). Nous n'avons pas utilisé d'autres seuils à grande échelle relativement à la SV : en choisissant une médiane, nous avons maximisé les chances de cerner les différences entre deux groupes égaux. Puisque ce seuil repose sur la distribution sous-jacente des scores, les comptes rendus des prévalences ne sont pas significatifs. Les comparaisons des prévalences entre groupes sont toutefois valides.

\section{Santé et fonctionnement}

Nous avons examiné l'autoévaluation de la santé générale des participants, par le biais de leur réponse à une seule question. Les catégories de réponse étaient " excellente ", " très bonne », " bonne », « passable » et « mauvaise».

Nous avons évalué les limitations fonctionnelles en demandant aux répondants dans quelle mesure leur trouble de l'humeur ou d'anxiété avait limité leurs activités au cours des 12 derniers mois dans 7 domaines : activités récréatives, loisirs et passe-temps; exercice ou sports; activités sociales en famille ou entre amis; tâches ménagères; magasinage et courses; voyages et vacances; hygiène personnelle ou habillement. Les répondants ont été répartis en trois groupes : ceux ayant déclaré n’avoir aucune limitation ou avoir peu de limitations fonctionnelles, ceux ayant déclaré avoir de nombreuses limitations dans une ou deux activités et ceux ayant déclaré avoir de nombreuses limitations dans trois activités ou plus.

\section{Comportements d'autogestion des troubles de l'humeur ou d'anxiété (activité physique, sommeil et méditation)}

Nous avons cerné les comportements liés à l'exercice en combinant les réponses à plusieurs questions. Les répondants devaient indiquer s'ils avaient commencé à faire de l'exercice pour gérer leur trouble de l'humeur ou d'anxiété, s'ils continuaient en faire et à quelle fréquence. Les catégories de comportements liés à la pratique d'exercices étaient : (1) participant ayant commencé à faire de l'exercice pour gérer son trouble à la suite de son diagnostic et qui a continué à en faire tous les jours; (2) participant ayant commencé à faire de l'exercice et ayant continué à en faire de quatre à six fois par semaine; (3) participant ayant commencé à faire de l'exercice et ayant continué à en faire de deux à trois fois par semaine ou moins; (4) participant ayant commencé à faire de l'exercice à la suite de son diagnostic mais ayant cessé d'en faire; (5) participant n'ayant jamais commencé à faire de l'exercice. Nous avons exclu des analyses portant sur l'activité physique les participants ayant déclaré n'avoir jamais commencé à faire de l'exercice après 
avoir reçu leur diagnostic car ils en faisaient déjà pour d'autres raisons $(\mathrm{n}=282)$.

Nous avons créé deux variables liées aux habitudes de sommeil en fonction des réponses sur le sommeil. On a demandé aux répondants s'ils avaient adopté de bonnes habitudes de sommeil (p. ex. respect d'un horaire de sommeil régulier) pour gérer leur trouble de l'humeur ou d'anxiété ( oui » ou « non ») et combien de temps ils passaient habituellement à dormir chaque nuit ("moins de 6 heures", " de 6 heures à moins de 9 heures " et « 9 heures et plus »).

Enfin, les répondants devaient indiquer s'ils prenaient des médicaments pour les aider à gérer leur trouble (" oui » ou «non »).

\section{Stress, adaptation et soutien social}

Nous avons mesuré le stress perçu à l'aide de la question suivante : " En pensant au niveau de stress dans votre vie, diriez-vous que la plupart de vos journées sont... "pas du tout stressantes"; "pas tellement stressantes"; "un peu stressantes”; "assez stressantes"; ou "extrêmement stressantes"? ». Dans le cadre de l'Enquête, deux questions portaient sur la capacité des répondants à faire face à des problèmes inattendus et difficiles ainsi qu'aux exigences de la vie quotidienne et ce sont ces réponses qui ont servi à mesurer la capacité d'adaptation des répondants. Nous avons considéré que la capacité d'adaptation des répondants était élevée chez ceux ayant obtenu un score de 8 ou plus aux deux questions combinées sur l'adaptation.

Le soutien social désigne les ressources qu'une personne reçoit d'autres personnes et qui améliorent son bien-être. Le soutien social peut comprendre à la fois un soutien tangible, comme de l'aide pour faire des activités et des ressources physiques, et un soutien intangible, comme de l'information, de l'affection ou un soutien affectif ${ }^{31}$. On a ajouté à l'EPMCC-TAH dix éléments tirés de l'Échelle de provisions sociales (EPS) ${ }^{32}$ : les répondants devaient préciser dans quelle mesure ils étaient d'accord avec chacun des énoncés (p. ex. " Il y a des personnes sur qui je peux compter pour m'aider en cas de réel besoin »). L'EPSS permet de mesurer les concepts d'alliance fiable, d'intégration sociale, d'encadrement et d'attachement. Les participants ayant obtenu un score moyen de 3 ou plus sur
l'EPS (score correspondant à des réponses se situant, en moyenne, à "d'accord » ou « fortement en accord ») ont été considérés comme ayant un niveau élevé de soutien social.

\section{Analyses statistiques}

Dans le cadre de cette étude, nous avons eu recours à l'analyse de données descriptives pour décrire le bien-être des personnes atteintes de troubles de l'humeur ou d'anxiété. Nous avons examiné le taux de prévalence de celles jugeant avoir un niveau de bien-être élevé en fonction des variables suivantes : groupe sociodémographique, type de trouble, santé et fonctionnement, comportements adoptés pour gérer le trouble de l'humeur ou d'anxiété, niveau de stress, capacité d'adaptation et soutien social. Nous avons aussi analysé la prévalence stratifiée selon le sexe afin de cerner une modification possible de l'effet. Nous avons réalisé une analyse de régression logistique afin d'étudier les associations entre le bien-être et a) les facteurs sociodémographiques, b) la santé et le fonctionnement, c) les comportements d'autogestion et d) le stress, l'adaptation et le soutien social. Nous avons conçu un ensemble de modèles hiérarchiques en ajoutant les blocs séquentiels de variables décrits plus haut. Toutes les analyses ont été réalisées à l'aide de l'application SAS Enterprise Guide, version 5.1 (SAS Institute, Cary, Caroline du Nord, É.-U.). Nous avons calculé la variance au moyen des poids obtenus par rééchantillonnage à répliques équilibrées à l'aide des procédures PROC SURVEY, ainsi que des poids fournis par Statistique Canada.

\section{Résultats}

Environ un répondant sur trois (31,4\%) a déclaré des scores d'AESM élevés. La proportion de répondants ayant reçu un diagnostic à la fois d'un trouble de l'humeur et d'un trouble d'anxiété et ayant déclaré un haut niveau de SV ou d'AESM était significativement moins élevée que celle de répondants atteints seulement de l'un de ces deux troubles (figure 1). Les scores moyens de SV étaient significativement plus élevés chez les personnes souffrant d'anxiété seulement (moyenne de 7,49, IC à $95 \%: 7,28$ à 7,70 ) que chez celles souffrant d'un trouble de l'humeur seulement (moyenne de 7,01, IC à $95 \%: 6,84$ à 7,18). Quant aux personnes souffrant de l'un ou de l'autre de ces troubles seulement, leurs scores de SV étaient significativement plus élevés que ceux des personnes atteintes de ces deux troubles (moyenne de 6,52, IC à $95 \%: 6,30$ à 6,74).

\section{Analyses bidimensionnelles}

\section{Facteurs sociodémographiques}

Les analyses bidimensionnelles (tableau 1) ont révélé une proportion plus élevée de personnes de 65 ans et plus ayant déclaré un haut niveau d'AESM (39,5\%), par comparaison avec les personnes de 35 à 49 ans $(26,3 \%)$. La proportion de personnes ayant déclaré un haut niveau de SV était plus élevée chez les personnes mariées ou vivant en union de fait $(52,7 \%)$ que chez les célibataires et personnes n'ayant jamais été mariées $(38,6 \%)$ ou que chez les personnes veuves, divorcées et séparées (36,8 \%). Aucune différence significative n'a été observée concernant le niveau de bien-être selon le sexe, le niveau de scolarité, le statut vis-à-vis de l'immigration ou le milieu de vie (urbain ou rural).

\section{Santé et fonctionnement}

La proportion de répondants déclarant un haut niveau d'AESM et de SV augmentait avec le niveau de santé générale autodéclarée. Une proportion significativement plus grande de personnes ayant déclaré n'avoir aucune limitation fonctionnelle ou très peu de limitations fonctionnelles déclarait un niveau de SV ou d'AESM plus élevé que celles ayant une à deux limitations ou trois limitations et plus. Les personnes ayant une à deux limitations jouissaient d'un bien-être supérieur à celles qui en avaient trois ou plus.

\section{Comportements d'autogestion}

La prévalence de niveaux élevés d'AESM et de SV était plus élevée chez les répondants ayant déclaré dormir de 6 à moins de 9 heures par nuit que chez ceux ayant déclaré dormir moins de 6 heures. Aucune différence significative n'a été observée parmi les personnes ayant déclaré avoir commencé à faire de la méditation ou à faire de l'exercice, ou ayant déclaré continuer à faire de l'exercice ou avoir adopté de bonnes habitudes de sommeil.

\section{Stress, adaptation et soutien social}

Le taux de prévalence de niveaux élevés d'AESM et de SV augmentait à mesure que le stress autodéclaré à l'égard de la vie diminuait (p. ex. 20,0 \% des répondants ayant indiqué que leur vie est " assez ou extrêmement stressante » ont déclaré un 
FIGURE 1

Proportion de Canadiens de 18 ans et plus souffrant d'un trouble de l'humeur ou d'anxiété qui ont présenté des scores élevés à l'autoévaluation de la santé mentale ou déclarant une grande satisfaction à l'égard de la vie, EPMCC-TAH de 2014

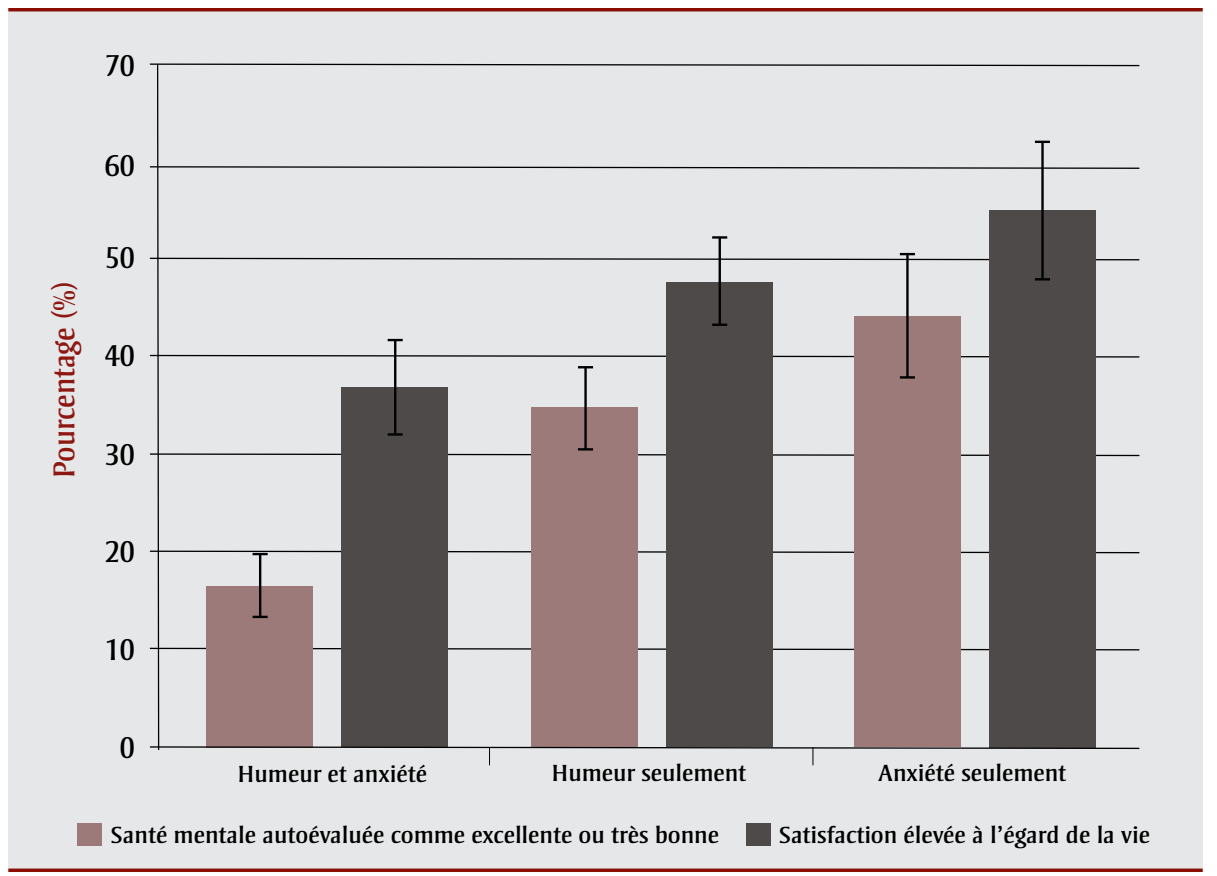

Abréviation : EPMCC-TAH, Enquête sur les personnes ayant une maladie chronique au Canada - Composante des troubles de l'humeur et d'anxiété.

Remarque : Les barres d'erreur représentent les intervalles de confiance à $95 \%$.

niveau élevé d'AESM contre 65,3 \% de ceux ayant indiqué que leur vie n'était " pas du tout stressante »). Une meilleure capacité d'adaptation était associée à un taux de prévalence accru d'AESM élevée et de SV élevée, par opposition à une capacité d'adaptation autodéclarée plus faible (pour l'AESM, 59,5 \% chez les personnes ayant une grande capacité d'adaptation contre $21,8 \%$ chez celles ayant une faible capacité d'adaptation et pour la SV, respectivement 61,9 \% contre 40,9 \%). De même, des niveaux de bien-être plus élevés ont été observés chez les personnes qui jugeaient bénéficier d'un grand soutien social par rapport à celles percevant leur degré de soutien social comme faible (respectivement $35,5 \%$ contre $14,3 \%$ pour l'AESM, et $51,8 \%$ contre $22,8 \%$ pour la SV).

Nous avons étudié la prévalence du bienêtre selon le sexe (données non présentées), mais sans constater de différences importantes dans les associations entre hommes et femmes.

\section{Régression logistique}

Les résultats produits par les modèles de régression logistique hiérarchique sont présentés dans le tableau 2 (AESM) et le tableau 3 (SV).

\section{Autoévaluation de la santé mentale}

Dans le modèle initial tenant compte des variables sociodémographiques seulement (modèle d'AESM 1), seuls l'âge et le revenu étaient significativement associés à un score d'AESM élevé (tableau 2). Les répondants de 65 ans et plus étaient presque deux fois plus enclins à présenter une AESM élevée que ceux de 35 à 49 ans. Les chances de présenter des scores d'AESM élevés augmentaient avec un revenu plus élevé. Létat matrimonial, le sexe, la scolarité, le statut vis-à-vis de l'immigration et le milieu de vie (urbain ou rural) ne constituaient pas des facteurs de prédiction significatifs d'une AESM élevée.

La probabilité d'obtenir des déclarations d'AESM élevée était significativement associée aux variables de santé et de fonctionnement ajoutées au modèle 2 . Plus particulièrement, les personnes ayant déclaré avoir une excellente santé étaient presque 13 fois plus enclines à présenter une AESM élevée, alors que les personnes se considérant en très bonne santé étaient 4 fois plus enclines à présenter une AESM élevée. Les répondants ayant déclaré n’avoir qu'une ou deux limitations fonctionnelles étaient plus susceptibles de présenter une AESM élevée que ceux ayant trois limitations ou plus. Enfin, les répondants souffrant d'un trouble de l'humeur seulement ou d'un trouble d'anxiété seulement étaient plus susceptibles de présenter une AESM élevée que ceux atteints de ces deux troubles. Après l'entrée des variables sur la santé, le revenu n'était plus significativement associé à une AESM élevée.

Les comportements d'autogestion ont été saisis dans le modèle 3. Aucune de ces variables n'était significativement associée à un score d'AESM élevé. Les variables de stress, d'adaptation et de soutien social ont été saisies dans le modèle 4 (sauf les comportements d'autogestion). Lorsque ces variables ont été intégrées au modèle, l'association entre le sexe et un score d'AESM élevé est devenue significative, les femmes étant 1,5 plus susceptibles de déclarer un score d'AESM élevé que les hommes. Lâge est demeuré une variable significative. Quant aux variables de santé et de fonctionnement, elles sont demeurées également significatives, sauf dans les cas où seul un trouble de l'humeur était présent. Les variables du stress, de l'adaptation et du soutien social étaient associées à un score d'AESM élevé. Une baisse du niveau de stress perçu augmentait les chances de présenter des scores d'AESM élevés. Les répondants ayant déclaré avoir de meilleures capacités d'adaptation et un plus grand soutien social étaient aussi plus susceptibles à présenter une AESM élevée.

\section{Satisfaction à l'égard de la vie}

Les résultats liés à la SV (tableau 3) se sont révélés à peu près semblables à ceux associés à l'AESM. Dans le modèle 1, les répondants plus jeunes (18 à 34 ans) et plus âgés (65 et plus) étaient associés à des probabilités plus élevées de déclarer une grande SV que ceux de 35 à 49 ans. Les personnes mariées ou vivant avec un conjoint de fait étaient plus susceptibles de déclarer une grande SV que les célibataires. Une association a été établie en fonction du revenu : les probabilités de déclarer une grande SV augmentaient avec un revenu plus élevé. Le modèle sociodémographique seul ne révélait aucune association significative avec le sexe, le niveau de scolarité, le statut vis-à-vis de l'immigration ou le milieu de vie (urbain ou rural). 
TABLEAU 1

Pourcentage de répondants présentant des résultats élevés à l'autoévaluation de la santé mentale (AESM) ou déclarant une grande satisfaction à l'égard de la vie, selon leurs caractéristiques sociodémographiques, leur état de santé et leur fonctionnement, l'autogestion et le stress, l'adaptation et le soutien social, Canada, EPMCC-TAH de 2014

\begin{tabular}{|c|c|c|}
\hline \multirow[t]{2}{*}{ Caractéristiques } & $\begin{array}{l}\text { Autoévaluation de la } \\
\text { santé mentale }\end{array}$ & Satisfaction à l'égard de la vie \\
\hline & $\begin{array}{l}\text { Niveau élevé } \\
\% \text { (IC à } 95 \%)\end{array}$ & $\begin{array}{l}\text { Niveau élevé } \\
\% \text { (IC à } 95 \%)\end{array}$ \\
\hline \multicolumn{3}{|l|}{ Sexe } \\
\hline Hommes & $29,9(24,9$ à 34,8) & $43,1(37,4$ à 48,7) \\
\hline Femmes & $32,2(29,1$ à 35,4$)$ & $48,0(44,4$ à 51,6$)$ \\
\hline \multicolumn{3}{|l|}{ Âge (ans) } \\
\hline 18 à 34 & $31,2(25,2$ à 37,1$)$ & $49,4(42,8$ à 55,9$)$ \\
\hline 35 à 49 & 26,3 (21,1 à 31,5) & $43,6(37,0$ à 50,1$)$ \\
\hline 50 à 64 & $32,2(27,8$ à 36,6$)$ & $43,3(38,1$ à 48,5) \\
\hline 65 et plus & $39,5(34,4$ à 44,6) & $51,5(46,1$ à 56,9$)$ \\
\hline \multicolumn{3}{|l|}{ État matrimonial } \\
\hline Célibataire/jamais marié & $27,2(22,2$ à 32,3) & 38,6 (32,9 à 44,4) \\
\hline Veuf/divorcé/séparé & 27,8 (22,8 à 32,7) & $36,8(31,0$ à 42,5$)$ \\
\hline Marié/union de fait & 34,4 (30,4 à 38,4) & $52,7(48,2$ à 57,2) \\
\hline \multicolumn{3}{|l|}{ Plus haut niveau de scolarité } \\
\hline Études secondaires non complétées & $31,1(23,2$ à 39,0$)$ & $43,2(34,0$ à 52,4$)$ \\
\hline Diplôme d'études secondaires & 30,3 (24,0 à 36,5) & 43,3 (36,3 à 50,2) \\
\hline $\begin{array}{l}\text { Études postsecondaires partielles ou } \\
\text { diplôme d'études postsecondaires }\end{array}$ & $31,4(28,3$ à 34,6$)$ & $47,2(43,5$ à 50,8) \\
\hline \multicolumn{3}{|l|}{$\begin{array}{l}\text { Quintile de suffisance du revenu du } \\
\text { ménage }\end{array}$} \\
\hline Q1 (quintile inférieur) & $23,5(19,1$ à 27,9$)$ & $33,9(28,2$ à 39,5$)$ \\
\hline Q2 & $31,0(25,2$ à 36,8$)$ & $37,6(31,2$ à 44,0) \\
\hline Q3 & $32,8(26,4$ à 39,2$)$ & 47,0 (39,9 à 54,2) \\
\hline Q4 & $32,4(26,2$ à 38,6) & $53,9(47,1$ à 60,8) \\
\hline Q5 (quintile supérieur) & $39,2(32,3$ à 46,0) & 61,6 (54,0 à 69,2) \\
\hline \multicolumn{3}{|l|}{ Immigrant } \\
\hline Oui & $30,0(20,9$ à 39,1$)$ & $46,4(35,7$ à 57,2$)$ \\
\hline Non & $31,6(28,8$ à 34,4$)$ & $46,2(43,0$ à 49,4) \\
\hline \multicolumn{3}{|l|}{ Milieu de vie } \\
\hline Urbain & $30,0(26,9$ à 33,1$)$ & 44,7 (41,2 à 48,2) \\
\hline Rural & 37,7 (32,5 à 42,9) & $53,1(48,2$ à 58,0$)$ \\
\hline \multicolumn{3}{|l|}{ Autoévaluation de la santé } \\
\hline Excellente & $70,1(60,4$ à 79,7) & $65,3(55,2$ à 75,4$)$ \\
\hline Très bonne & 45,3 (39,5 à 51,1) & $60,8(54,0$ à 67,5$)$ \\
\hline Bonne & 23,6 (19,9 à 27,4) & $44,1(39,3$ à 48,8) \\
\hline Passable & 13,6 (9,9 à 17,3) & 28,5 (22,5 à 34,4) \\
\hline Mauvaise & $12,8(7,6 \text { à } 17,9)^{\mathrm{a}}$ & $18,0(10,5 \text { à } 25,4)^{a}$ \\
\hline \multicolumn{3}{|l|}{ Limitations fonctionnelles } \\
\hline Aucune ou peu de limitations & 41,0 (37,3 à 44,6) & $53,4(49,3$ à 57,5) \\
\hline 1 à 2 limitations & $21,0(15,2$ à 26,9$)$ & 42,7 (36,2 à 49,3) \\
\hline 3 limitations ou plus & $7,9(4,7 \text { à } 11,1)^{a}$ & $24,1(18,0$ à 30,3$)$ \\
\hline
\end{tabular}

Suite page suivante
Lorsque les variables de la santé et du fonctionnement ont été prises en compte (modèle 2), les probabilités de déclarer une grande SV augmentaient avec une meilleure autoévaluation de la santé. Les répondants ayant déclaré moins de limitations fonctionnelles avaient de meilleures chances de déclarer une grande SV que ceux ayant trois limitations ou plus. Contrairement au modèle de prédiction de l'AESM, aucune différence significative n'a été observée entre les résultats des personnes souffrant d'un trouble d'anxiété seulement, ceux des personnes souffrant d'un trouble de l'humeur seulement et ceux des personnes atteintes de ces deux troubles. Lorsque les variables de la santé et du fonctionnement ont été intégrées, la plupart des associations avec le revenu n'étaient plus significatives, à l'exception des probabilités de déclarer une grande SV, lesquelles étaient plus élevées dans le groupe affichant les revenus les plus élevés par rapport aux autres groupes.

Nous n’avons observé aucune association significative entre la satisfaction à l'égard de la vie et les comportements d'autogestion lorsque ces variables ont été ajoutées au modèle (modèle 3). Toutefois, toutes les variables liées au stress, à l'adaptation et au soutien social donnaient lieu à des associations significatives (modèle 4). Les répondants qui déclaraient éprouver moins de stress dans leur vie avaient plus de chances de déclarer une grande SV que ceux qui atteignaient le plus haut niveau de stress dans leur vie. Ceux qui ont déclaré avoir de meilleures capacités d'adaptation et jouir d'un plus grand soutien social avaient significativement plus de chances de déclarer une grande SV que les répondants ayant peu de capacités d'adaptation et déclarant recevoir peu de soutien social. Lorsque les variables du stress, de l'adaptation et du soutien social ont été ajoutées, on a observé que les femmes étaient significativement plus susceptibles de déclarer une grande SV que les hommes. L'état matrimonial et le revenu (pour le groupe des revenus les plus élevés seulement) sont demeurés des variables significatives dans ce modèle.

\section{Analyse}

Globalement, un tiers des Canadiens ayant déclaré avoir reçu un diagnostic de trouble de l'humeur et presque la moitié des Canadiens souffrant d'un trouble d'anxiété ont évalué que leur santé mentale était très 
TABLEAU 1 (suite)

Pourcentage de répondants présentant des résultats élevés à l'autoévaluation de la santé mentale (AESM) ou déclarant une grande satisfaction à l'égard de la vie, selon leurs caractéristiques sociodémographiques, leur état de santé et leur fonctionnement, l'autogestion et le stress, l'adaptation et le soutien social, Canada, EPMCC-TAH de 2014

\begin{tabular}{|c|c|c|}
\hline \multirow[t]{2}{*}{ Caractéristiques } & $\begin{array}{l}\text { Autoévaluation de la } \\
\text { santé mentale }\end{array}$ & Satisfaction à l'égard de la vie \\
\hline & $\begin{array}{l}\text { Niveau élevé } \\
\% \text { (IC à } 95 \%)\end{array}$ & $\begin{array}{l}\text { Niveau élevé } \\
\% \text { (IC à } 95 \%)\end{array}$ \\
\hline \multicolumn{3}{|l|}{ Type de trouble } \\
\hline Humeur et anxiété & $16,4(13,1$ à 19,6$)$ & $36,9(31,9$ à 41,9$)$ \\
\hline Humeur seulement & $34,7(30,5$ à 38,9$)$ & 47,8 (43,2 à 52,4) \\
\hline Anxiété seulement & $44,2(37,9$ à 50,5$)$ & $55,0(48,1$ à 62,0$)$ \\
\hline \multicolumn{3}{|l|}{ Exercice } \\
\hline $\begin{array}{l}\text { A commencé à faire de l'exercice, puis } \\
\text { a continué tous les jours }\end{array}$ & $30,8(23,7$ à 37,9$)$ & $44,7(36,1$ à 53,3$)$ \\
\hline $\begin{array}{l}\text { A commencé à faire de l'exercice, puis } \\
\text { a continué } 4 \text { à } 6 \text { fois par semaine }\end{array}$ & $43,0(34,5$ à 51,5$)$ & $59,7(51,0$ à 68,3) \\
\hline $\begin{array}{l}\text { A commencé à faire de l'exercice, puis } \\
\text { a continué } 2 \text { à } 3 \text { fois par semaine ou } \\
\text { moins }\end{array}$ & $29,2(23,5$ à 35,0$)$ & $51,8(45,2$ à 58,4$)$ \\
\hline $\begin{array}{l}\text { A commencé à faire de l'exercice, mais } \\
\text { a cessé d'en faire }\end{array}$ & $29,4(23,1$ à 35,8$)$ & $40,0(33,1$ à 46,9) \\
\hline $\begin{array}{l}\text { N'a jamais commencé à faire de } \\
\text { l'exercice }\end{array}$ & $30,2(25,9$ à 34,6$)$ & $41,2(36,5$ à 45,8) \\
\hline \multicolumn{3}{|c|}{ A adopté de bonnes habitudes de sommeil après avoir reçu le diagnostic } \\
\hline Oui & $30,0(26,9$ à 33,2$)$ & $45,2(41,7$ à 48,6) \\
\hline Non & $34,5(29,5$ à 39,4) & $48,7(43,0$ à 54,3$)$ \\
\hline \multicolumn{3}{|c|}{ Nombre d'heures de sommeil (habituellement) par nuit } \\
\hline Moins de 6 heures & $20,6(15,5$ à 25,8$)$ & $32,4(25,4$ à 39,3$)$ \\
\hline 6 heures à moins de 9 heures & $34,6(31,2$ à 38,0$)$ & $50,2(46,4$ à 54,1$)$ \\
\hline 9 heures ou plus & $30,2(23,7$ à 36,8$)$ & $45,0(38,1$ à 51,9$)$ \\
\hline \multicolumn{3}{|c|}{ Recours à la méditation comme mesure d'autogestion de la maladie } \\
\hline Oui & $31,4(27,4$ à 35,4$)$ & $46,3(42,0$ à 50,6$)$ \\
\hline Non & $31,3(27,8$ à 34,8$)$ & $46,1(41,9$ à 50,3$)$ \\
\hline \multicolumn{3}{|l|}{ Stress perçu (journées) } \\
\hline Pas du tout stressantes & $65,3(51,3$ à 79,2) & $61,7(48,2$ à 75,2$)$ \\
\hline Pas tellement stressantes & $46,8(40,8$ à 52,9$)$ & $61,4(55,1$ à 67,6$)$ \\
\hline Un peu stressantes & $33,1(29,0$ à 37,2$)$ & 49,2 (44,4 à 53,9) \\
\hline Assez ou extrêmement stressantes & $20,0(16,4$ à 23,6$)$ & $35,2(30,4$ à 39,9) \\
\hline \multicolumn{3}{|l|}{ Adaptation } \\
\hline Grande capacité d'adaptation & $59,5(53,6$ à 65,5$)$ & $61,9(56,0$ à 67,7$)$ \\
\hline Faible capacité d'adaptation & $21,8(19,1$ à 24,5$)$ & 40,9 (37,4 à 44,4) \\
\hline \multicolumn{3}{|l|}{ Soutien social perçu } \\
\hline Grand soutien social & $35,5(32,4$ à 38,6$)$ & $51,8(48,2$ à 55,3$)$ \\
\hline Peu de soutien social & $14,3(10,6$ à 18,0$)$ & $22,8(17,6$ à 28,1$)$ \\
\hline
\end{tabular}

Abréviations : EPMCC-TAH, Enquête sur les personnes ayant une maladie chronique au Canada - Composante des troubles de I'humeur et d'anxiété; IC, intervalle de confiance; Q, quintile.

Remarques : Les résultats à l'autoévaluation de la santé mentale (AESM) ont été considérés comme élevés si les répondants jugeaient que leur santé mentale était très bonne ou excellente. La satisfaction à l'égard de la vie (SV) a été considérée comme élevée lorsque les participants ont attribué à cette composante un score de 8 ou plus sur une échelle de 0 à 10 .

${ }^{a}$ Faire preuve de prudence lors de l'interprétation de ces résultats car leur coefficient de variation se situe entre 16,6\% et 33,3\%. bonne ou excellente. Toutefois, seulement $16 \%$ des personnes souffrant de ces deux troubles ont enregistré un tel score d'AESM. Les niveaux de satisfaction à l'égard de la vie étaient aussi assez élevés, avec un score moyen de 6,98 pour l'ensemble de l'échantillon, et supérieur chez les répondants ayant déclaré n'être atteint que d'un trouble de l'humeur $(7,01)$ ou que d'un trouble d'anxiété $(7,49)$. La prévalence d'une grande SV était significativement plus faible chez les répondants ayant déclaré être atteint à la fois d'un trouble d'anxiété et d'un trouble de l'humeur par rapport aux répondants ayant déclaré n'être atteint que de l'un de ces troubles.

Les analyses bidimensionnelles ont révélé que la prévalence d'une AESM ou d'une SV élevée variait selon les caractéristiques sociodémographiques et l'état de santé, le fonctionnement, le stress, les capacités d'adaptation et le soutien social. Quant aux comportements d'autogestion, des différences significatives n'ont cependant été observées qu'à propos des heures de sommeil.

Une proportion significativement plus grande d'adultes plus âgés (65 et plus) a enregistré des scores d'AESM plus élevés par rapport aux adultes de 35 à 49 ans. Cette relation était aussi marquée dans les modèles multivariés concernant l'AESM et la SV. Toutefois, avec l'intégration des variables du stress, de l'adaptation et du soutien social dans le modèle de SV, cette relation n'était plus significative. Bien que la littérature laisse entendre que les degrés de maladie mentale et de détresse psychologique seraient plus faibles chez les adultes plus âgés ${ }^{33}$, il est intéressant de constater que même chez les adultes souffrant d'un trouble de l'humeur ou d'anxiété, un âge plus avancé est associé à des degrés de bien-être supérieurs. Il convient donc de déterminer si ces faits sont attribuables à l'âge ou à un effet de cohorte.

Les femmes étaient plus susceptibles que les hommes de présenter des scores élevés d'AESM ou de SV lorsque les variables du stress, de l'adaptation et du soutien social étaient intégrées au modèle. Un revenu plus élevé était associé à de plus grandes probabilités de SV et de meilleurs scores d'AESM, ce qui cadre avec les études montrant qu'un revenu plus élevé est associé à un bien-être subjectif supérieur ${ }^{34}$. Une fois les variables sur la santé ajoutées (modèle 2), ces relations s'atténuaient 
TABLEAU 2

Modèles de régression logistique pour la prédiction de résultats élevés à l'autoévaluation de la santé mentale (AESM) chez les Canadiens de 18 ans et plus atteints d'un trouble de l'humeur ou d'anxiété

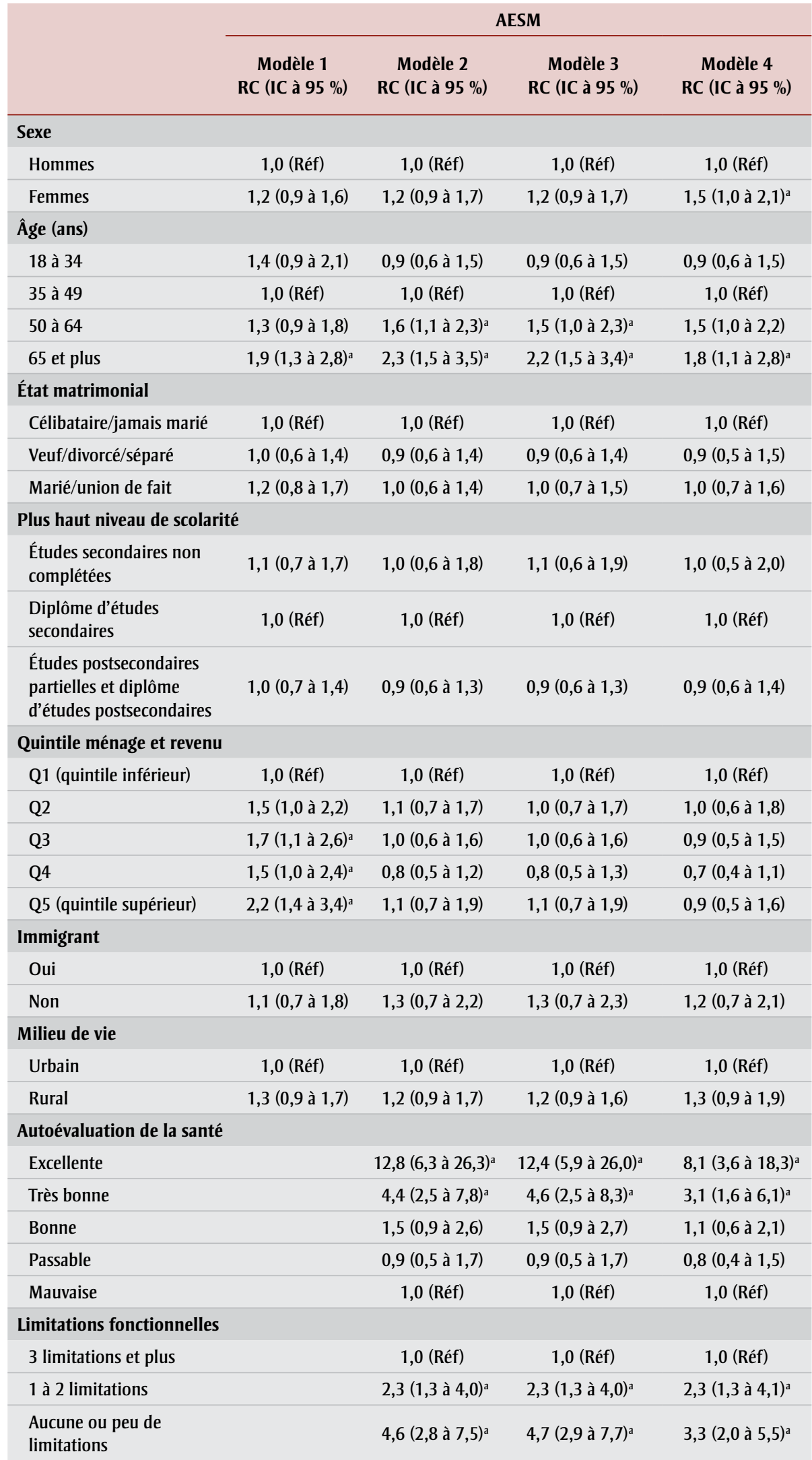

Suite page suivante concernant la SV et n'étaient plus significatives pour l'AESM. En raison de la relation réciproque entre les variables de la santé et du revenu, il est important d'étudier cette relation dans de futures recherches. Le niveau de scolarité n'était pas associé à un bien-être supérieur au sein de cette population, ce qui pourrait s'expliquer par le fait que les effets du niveau de scolarité étaient médiés par le revenu. L'ajout de ces deux variables dans le modèle a donc produit des résultats non significatifs en ce qui concerne la scolarité.

Les personnes mariées ou en union de fait étaient plus susceptibles de déclarer une grande SV que les célibataires et les personnes jamais mariées, les veufs et les divorcés. Ceci cadre avec la littérature, qui indique que le célibat, la séparation, le divorce et le veuvage sont associés à des résultats de santé mentale médiocres ${ }^{35}$. Il semblerait que ce n'est pas tant la relation que la qualité de la relation qui constitue le meilleur facteur de prédiction du bienêtre $^{36}$. Les associations significatives que nous avons constatées à propos du soutien social appuient également cette idée.

Par opposition aux conclusions antérieures qui ont mis en évidence les bienfaits de l'exercice pour améliorer l'humeur et le bien-être des personnes souffrant de dépression ou de divers troubles d'anxiétée-12, le fait de commencer à faire de l'exercice ou de poursuivre ses activités physiques ne s'est pas révélé au sein de notre échantillon comme un comportement d'autogestion associé à des niveaux de bien-être significativement plus élevés. Cependant, la mesure utilisée dans le cadre de notre étude n'englobait pas les niveaux réels d'activité physique, et nous avons exclu les répondants qui avaient déclaré faire déjà de l'exercice. Les travaux de recherche à venir devront utiliser des mesures plus rigoureuses liées à l'activité physique, à la fois les niveaux d'activité physique et les motifs amenant à pratiquer une activité physique. Les répondants qui dormaient de 6 heures à moins de 9 heures par nuit ont déclaré des niveaux de bien-être plus grands que ceux qui dormaient 6 heures ou moins, mais cette relation ne s'est pas maintenue dans les modèles de régression logistique.

Fait plutôt surprenant compte tenu de sa popularité dans les traitements en santé mentale, la méditation ne s'est pas révélée un facteur de prédiction significatif du 
TABLEAU 2 (suite)

Modèles de régression logistique pour la prédiction de résultats élevés à l'autoévaluation de la santé mentale (AESM) chez les Canadiens de 18 ans et plus atteints d'un trouble de I'humeur ou d'anxiété

\begin{tabular}{|c|c|c|c|c|}
\hline & \multicolumn{4}{|c|}{ AESM } \\
\hline & $\begin{array}{c}\text { Modèle } 1 \\
\text { RC (IC à } 95 \%)\end{array}$ & $\begin{array}{c}\text { Modèle } 2 \\
\text { RC (IC à } 95 \% \text { ) }\end{array}$ & $\begin{array}{c}\text { Modèle } 3 \\
\text { RC (IC à } 95 \% \text { ) }\end{array}$ & $\begin{array}{c}\text { Modèle } 4 \\
\text { RC (IC à } 95 \%)\end{array}$ \\
\hline \multicolumn{5}{|l|}{ Type de trouble } \\
\hline Humeur et anxiété & & 1,0 (Réf) & 1,0 (Réf) & 1,0 (Réf) \\
\hline Humeur seulement & & $1,7(1,2 \text { à } 2,3)^{\mathrm{a}}$ & $1,7(1,2 \text { à } 2,3)^{a}$ & $1,4(0,9$ à 1,9$)$ \\
\hline Anxiété seulement & & $2,3(1,5 \text { à } 3,5)^{a}$ & $2,4(1,6 \text { à } 3,6)^{a}$ & $2,1(1,3 \text { à } 3,2)^{\mathrm{a}}$ \\
\hline \multicolumn{5}{|c|}{ Recours à l'exercice comme mesure d'autogestion de la maladie } \\
\hline $\begin{array}{l}\text { A commencé à faire de } \\
\text { l'exercice, puis a } \\
\text { continué tous les jours }\end{array}$ & & & $0,9(0,5$ à 1,5$)$ & \\
\hline $\begin{array}{l}\text { A commencé à faire de } \\
\text { l'exercice, puis a } \\
\text { continué } 4 \text { à } 6 \text { fois par } \\
\text { semaine }\end{array}$ & & & $1,1(0,6$ à 1,9$)$ & \\
\hline $\begin{array}{l}\text { A commencé à faire de } \\
\text { l'exercice, puis a } \\
\text { continué } 2 \text { à } 3 \text { fois par } \\
\text { semaine ou moins }\end{array}$ & & & $0,7(0,4$ à 1,2$)$ & \\
\hline $\begin{array}{l}\text { A commencé à faire de } \\
\text { l'exercice, mais a cessé } \\
\text { d'en faire }\end{array}$ & & & 1,0 (Réf) & \\
\hline $\begin{array}{l}\text { N'a jamais commencé à } \\
\text { faire de l'exercice }\end{array}$ & & & $0,8(0,5$ à 1,4$)$ & \\
\hline \multicolumn{5}{|c|}{ A adopté de bonnes habitudes de sommeil après avoir reçu le diagnostic } \\
\hline Oui & & & $0,9(0,7$ à 1,3$)$ & \\
\hline Non & & & 1,0 (Réf) & \\
\hline \multicolumn{5}{|c|}{ Nombre d'heures de sommeil (habituellement) par nuit } \\
\hline Moins de 6 heures & & & 1,0 (Réf) & \\
\hline $\begin{array}{l}6 \text { heures à moins de } 9 \\
\text { heures }\end{array}$ & & & $1,0(0,7$ à 1,6$)$ & \\
\hline 9 heures ou plus & & & $1,3(0,7$ à 2,2) & \\
\hline \multicolumn{5}{|c|}{ Recours à la méditation comme mesure d'autogestion de la maladie } \\
\hline Oui & & & $1,2(0,9$ à 1,7$)$ & \\
\hline Non & & & 1,0 (Réf) & \\
\hline \multicolumn{5}{|l|}{ Stress perçu (journées) } \\
\hline Pas du tout stressantes & & & & $4,3(1,9 \text { à } 9,8)^{a}$ \\
\hline Pas tellement stressantes & & & & $2,4(1,6 \text { à } 3,6)^{\mathrm{a}}$ \\
\hline Un peu stressantes & & & & $1,7(1,2 \text { à } 2,4)^{a}$ \\
\hline $\begin{array}{l}\text { Assez ou extrêmement } \\
\text { stressantes }\end{array}$ & & & & 1,0 (Réf) \\
\hline
\end{tabular}

Suite page suivante

bien-être au sein de notre échantillon. Il est difficile d'avancer des hypothèses à ce sujet, puisque nous n'avons pas de précisions quant au type de méditation pratiqué, à la fréquence et à la durée des séances ou à la formation associés aux exercices de méditation des participants. Nos résultats ne sont toutefois pas contradictoires avec ceux de la littérature, qui présente des résultats divers sur l'incidence de la méditation sur le bien-être ${ }^{18}$. Notre étude décrit un échantillon représentatif de la population canadienne souffrant de troubles de l'humeur ou d'anxiété et pratiquant une forme de méditation qu'ils jugent efficace dans un cadre de vie normal. Il s'agit là d'une contribution importante en la matière à la littérature. Il convient toutefois de réaliser des recherches plus poussées avant d'en tirer des conclusions.

Dans les modèles de régression logistique multivariés, un stress moins important, un meilleur soutien social et de meilleures capacités d'adaptation étaient significativement associés à de meilleures probabilités de bien-être. Puisque les capacités d'adaptation englobent souvent des stratégies qui concordent avec les aptitudes mises en valeur dans les séances de traitement ${ }^{19}$, il serait raisonnable de penser que les stratégies d'adaptation favorisent le bien-être. De même, les personnes ayant estimé qu'elles bénéficiaient d'un grand soutien social étaient plus susceptibles de présenter des résultats d'AESM et de SV élevés, ce qui cadre avec les conclusions de la littérature sur le bien-être chez les personnes souffrant de troubles de l'humeur ou d'anxiété 25,26 .

\section{Points forts et limites}

Cette étude porte sur le bien-être au sein d'un échantillon représentatif de la population canadienne à domicile atteinte d'un trouble de l'humeur ou d'anxiété. Elle fournit une description utile sur la prévalence du bien-être et sur la relation entre bienêtre et comportements d'autogestion, niveaux de stress, capacités d'adaptation et degré de soutien social au sein de cette population. Cet échantillon diffère de ceux utilisés dans la plupart des autres études sur le sujet, qui ont plutôt tendance à se concentrer sur de petits échantillons cliniques. De plus, la plupart des études sur la santé mentale portent sur les troubles mentaux et les dysfonctions, alors que la compréhension des corrélats de la santé mentale positive chez les personnes atteintes de troubles mentaux peut fournir des indices importants sur leur potentiel de rétablissement : les résultats de cette étude révèlent qu'il est possible d'atteindre un haut niveau de bien-être même aux prises avec un problème de santé mentale.

Il nous faut cependant faire état de plusieurs limitations significatives. Nous n'avons aucune indication précise sur la situation actuelle des troubles de l'humeur ou d'anxiété dont souffrent les répondants. Lorsque le bien-être est étudié, l'idéal serait de déterminer quels répondants présentent encore des symptômes et lesquels ont vécu un épisode déjà résolu. Nous avons utilisé les limitations fonctionnelles liées aux troubles de l'humeur ou d'anxiété comme 
TABLEAU 2 (suite)

Modèles de régression logistique pour la prédiction de résultats élevés à l'autoévaluation de la santé mentale (AESM) chez les Canadiens de 18 ans et plus atteints d'un trouble de I'humeur ou d'anxiété

\begin{tabular}{|c|c|c|c|c|}
\hline & \multicolumn{4}{|c|}{ AESM } \\
\hline & $\begin{array}{c}\text { Modèle } 1 \\
\text { RC (IC à } 95 \%)\end{array}$ & $\begin{array}{c}\text { Modèle } 2 \\
\text { RC (IC à } 95 \% \text { \%) }\end{array}$ & $\begin{array}{c}\text { Modèle } 3 \\
\text { RC (IC à } 95 \% \text { \%) }\end{array}$ & $\begin{array}{c}\text { Modèle } 4 \\
\text { RC (IC à } 95 \% \text { ) }\end{array}$ \\
\hline \multicolumn{5}{|l|}{ Adaptation } \\
\hline $\begin{array}{l}\text { Grande capacité } \\
\text { d'adaptation }\end{array}$ & & & & $4,2(3,0 \text { à } 6,0)^{a}$ \\
\hline $\begin{array}{l}\text { Faible capacité } \\
\text { d'adaptation }\end{array}$ & & & & 1,0 (Réf) \\
\hline \multicolumn{5}{|l|}{ Soutien social perçu } \\
\hline Grand soutien social & & & & $1,7(1,1 \text { à } 2,7)^{a}$ \\
\hline Peu de soutien social & & & & 1,0 (Réf) \\
\hline
\end{tabular}

Abréviations : AESM, autoévaluation de la santé mentale; IC, intervalle de confiance; Q, quintile; RC, rapport de cotes; Réf, groupe de référence.

Remarques : Les résultats à l'AESM ont été considérés comme élevés si les répondants jugeaient que leur santé mentale était très bonne ou excellente.

Le modèle 1 est le modèle de départ englobant seulement les variables sociodémographiques. Le modèle 2 intègre les variables sociodémographiques et les variables de santé et de fonctionnement. Le modèle 3 intègre ces variables ainsi que les comportements d'autogestion. Le modèle 4 intègre les variables sociodémographiques, les variables de santé et de fonctionnement et les variables du stress, de l'adaptation et du soutien social, mais n'inclut pas les variables d'autogestion.

${ }^{a}$ Statistiquement significatif à $p<0,05$.

TABLEAU 3

Modèles de régression logistique pour la prédiction de résultats de grande satisfaction à l'égard de la vie (SV) chez les Canadiens de 18 ans et plus atteints d'un trouble anxieux ou de l'humeur

\begin{tabular}{|c|c|c|c|c|}
\hline & \multicolumn{4}{|c|}{ SV } \\
\hline & $\begin{array}{c}\text { Modèle } 1 \\
\text { RC (IC à } 95 \% \text { ) }\end{array}$ & $\begin{array}{c}\text { Modèle } 2 \\
\text { RC (IC à } 95 \%)\end{array}$ & $\begin{array}{c}\text { Modèle } 3 \\
\text { RC (IC à } 95 \% \text { ) }\end{array}$ & $\begin{array}{c}\text { Modèle } 4 \\
\text { RC (IC à } 95 \%)\end{array}$ \\
\hline \multicolumn{5}{|l|}{ Sexe } \\
\hline Hommes & 1,0 (Réf) & 1,0 (Réf) & 1,0 (Réf) & 1,0 (Réf) \\
\hline Femmes & $1,3(1,0$ à 1,8$)$ & 1,3 (1,0 à 1,8) & $1,3(1,0$ à 1,8$)$ & $1,4(1,0 \text { à } 2,0)^{a}$ \\
\hline \multicolumn{5}{|l|}{ Âge (ans) } \\
\hline 18 à 34 & $1,8(1,2 \text { à } 2,9)^{\mathrm{a}}$ & $1,6(1,0$ à 2,5$)$ & 1,5 (0,9 à 2,5) & $1,6(1,0$ à 2,6$)$ \\
\hline 35 à 49 & 1,0 (Réf) & 1,0 (Réf) & 1,0 (Réf) & 1,0 (Réf) \\
\hline 50 à 64 & $1,0(0,7$ à 1,5$)$ & 1,2 (0,8 à 1,7) & 1,1 (0,8 à 1,7) & 1,1 (0,7 à 1,6) \\
\hline 65 et plus & $1,6(1,1 \text { à } 2,3)^{\mathrm{a}}$ & $1,7(1,1 \text { à } 2,6)^{\mathrm{a}}$ & $1,7(1,1 \text { à } 2,6)^{a}$ & $1,4(0,9$ à 2,1$)$ \\
\hline \multicolumn{5}{|l|}{ État matrimonial } \\
\hline Célibataire/jamais marié & 1,0 (Réf) & 1,0 (Réf) & 1,0 (Réf) & 1,0 (Réf) \\
\hline Veuf/divorcé/séparé & 1,2 (0,8 à 1,9) & 1,2 (0,8 à 1,9) & $1,2(0,8$ à 1,9) & $1,3(0,8$ à 2,0$)$ \\
\hline Marié/union de fait & $1,8(1,2 \text { à } 2,7)^{\mathrm{a}}$ & $1,7(1,1 \text { à } 2,5)^{\mathrm{a}}$ & $1,7(1,1 \text { à } 2,6)^{a}$ & $1,8(1,2 \text { à } 2,8)^{a}$ \\
\hline \multicolumn{5}{|l|}{ Plus haut niveau de scolarité } \\
\hline $\begin{array}{l}\text { Études secondaires non } \\
\text { complétées }\end{array}$ & $1,2(0,7$ à 2,1) & $1,2(0,7$ à 2,1$)$ & $1,2(0,7$ à 2,1$)$ & 1,5 (0,8 à 2,6) \\
\hline Diplôme d'études secondaires & 1,0 (Réf) & 1,0 (Réf) & 1,0 (Réf) & 1,0 (Réf) \\
\hline $\begin{array}{l}\text { Études postsecondaires } \\
\text { partielles et diplôme d'études } \\
\text { postsecondaires }\end{array}$ & 0,9 (0,7 à 1,3) & 0,9 (0,6 à 1,3) & 0,9 (0,6 à 1,2) & 0,9 (0,6 à 1,3) \\
\hline
\end{tabular}

Suite page suivante indicateurs indirects de la situation vis-àvis de la maladie, mais cette mesure n'est pas parfaite.

Les problèmes de sommeil, notamment les périodes de sommeil plus ou moins longues par rapport aux nombres d'heures de sommeil habituelles, figurent parmi les critères de diagnostic des troubles dépressifs majeurs (TDM) ${ }^{13}$. Il est possible que les différences observées, dans le cadre d'analyses univariées, sur le plan du bien-être en fonction de nombre d'heures de sommeil soient fonction de l'épisode de TDM alors en cours.

Le fait qu'il s'agisse d'une enquête transversale ne nous autorise pas à examiner la dimension chronologique des événements : il est impossible de déterminer si c'est le bien-être qui a une influence sur les caractéristiques du stress, de l'adaptation et du soutien social ou si ce sont ces derniers qui ont une influence sur le bien-être. Les deux cas de figure sont dans une certaine mesure envisageables. Des études longitudinales et expérimentales pourraient contribuer à traiter de ces questions. Il est cependant utile de comprendre ces relations au sein d'un large échantillon représentatif de la population, puisqu'elles permettent d'évaluer la généralisabilité des observations tirées d'études de moindre envergure.

Enfin, s'il est vrai que les biais liés aux non-réponses peuvent être à l'origine de certaines des relations observées, l'absence totale de relations significatives entre les comportements d'autogestion et le bienêtre laisse entendre que ce n'est pas le cas. (Autrement dit, si les constatations significatives découlaient seulement d'un biais lié aux non-réponses, la relation entre les comportements d'autogestion et le bienêtre aurait été positive et significative.)

\section{Conclusion}

Lorsque nous avons inclus dans les modèles de régression logistique les caractéristiques sociodémographiques, l'état de santé, le fonctionnement, le stress, l'adaptation et le soutien social, nous avons constaté qu'aucun des comportements d'autogestion n'était significativement associé à un bienêtre supérieur, contrairement aux variables $\mathrm{du}$ stress, de l'adaptation et du soutien social. Cette constatation laisse penser que des stratégies d'adaptation saines et la perception d'un grand soutien social seraient primordiales chez les Canadiens atteints de 
TABLEAU 3 (suite)

Modèles de régression logistique pour la prédiction de résultats de grande satisfaction à l'égard de la vie (SV) chez les Canadiens de 18 ans et plus atteints d'un trouble anxieux ou de l'humeur

\begin{tabular}{|c|c|c|c|c|}
\hline & \multicolumn{4}{|c|}{ SV } \\
\hline & $\begin{array}{c}\text { Modèle } 1 \\
\text { RC (IC à } 95 \%) \\
\end{array}$ & $\begin{array}{c}\text { Modèle } 2 \\
\text { RC (IC à } 95 \%)\end{array}$ & $\begin{array}{c}\text { Modèle } 3 \\
\text { RC (IC à } 95 \%) \\
\end{array}$ & $\begin{array}{c}\text { Modèle } 4 \\
\text { RC (IC à } 95 \% \text { ) }\end{array}$ \\
\hline \multicolumn{5}{|l|}{ Quintile ménage et revenu } \\
\hline Q1 (quintile inférieur) & 1,0 (Réf) & 1,0 (Réf) & 1,0 (Réf) & 1,0 (Réf) \\
\hline Q2 & $1,1(0,7$ à 1,6$)$ & 0,8 (0,5 à 1,3) & 0,8 (0,5 à 1,3) & $0,8$ (0,5 à 1,3$)$ \\
\hline Q3 & $1,8(1,2 \text { à } 2,7)^{\mathrm{a}}$ & $1,2(0,7$ à 1,9$)$ & $1,2(0,7$ à 1,9$)$ & $1,1(0,7$ à 1,9$)$ \\
\hline Q4 & $2,2(1,5$ à 3,4) & 1,5 (1,0 à 2,4) & 1,5 (0,9 à 2,4) & 1,5 (0,9 à 2,4) \\
\hline Q5 (quintile supérieur) & $3,1\left(1,9\right.$ à 4,9) ${ }^{\mathrm{a}}$ & $2,0(1,2 \text { à } 3,4)^{a}$ & $2,0(1,2 \text { à } 3,3)^{\mathrm{a}}$ & $1,9(1,1 \text { à } 3,4)^{\mathrm{a}}$ \\
\hline \multicolumn{5}{|l|}{ Immigrant } \\
\hline Oui & 1,0 (Réf) & 1,0 (Réf) & 1,0 (Réf) & 1,0 (Réf) \\
\hline Non & $1,0(0,6$ à 1,7$)$ & $1,0$ (0,6 à 1,8$)$ & $1,1(0,6$ à 1,8) & $1,0(0,6$ à 1,7$)$ \\
\hline \multicolumn{5}{|l|}{ Milieu de vie } \\
\hline Urbain & 1,0 (Réf) & 1,0 (Réf) & 1,0 (Réf) & 1,0 (Réf) \\
\hline Rural & 1,2 (0,9 à 1,6) & $1,2(0,9$ à 1,6$)$ & 1,2 (0,9 à 1,6) & 1,3 (1,0 à 1,7) \\
\hline \multicolumn{5}{|l|}{ Autoévaluation de la santé } \\
\hline Excellente & & $5,4(2,5 \text { à } 11,7)^{a}$ & $4,7(2,1 \text { à } 10,6)^{\mathrm{a}}$ & $3,2(1,4 \text { à } 7,4)^{a}$ \\
\hline Très bonne & & $4,7(2,5 \text { à 9,1) })^{a}$ & $4,3(2,2 \text { à } 8,3)^{a}$ & $3,2(1,6 \text { à } 6,4)^{a}$ \\
\hline Bonne & & $2,6(1,4 \text { à } 4,7)^{a}$ & $2,4(1,3 \text { à } 4,5)^{a}$ & 1,9 (1,0 à 3,6) \\
\hline Passable & & 1,7 (0,9 à 3,2) & $1,6(0,9$ à 3,0) & 1,4 (0,7 à 2,7) \\
\hline Mauvaise & & 1,0 (Réf) & 1,0 (Réf) & 1,0 (Réf) \\
\hline \multicolumn{5}{|l|}{ Limitations fonctionnelles } \\
\hline 3 limitations et plus & & 1,0 (Réf) & 1,0 (Réf) & 1,0 (Réf) \\
\hline 1 à 2 limitations & & $1,9(1,2 \text { à } 3,0)^{a}$ & $2,0(1,2$ à 3,1) & $1,8(1,1 \text { à } 3,0)^{\mathrm{a}}$ \\
\hline Aucune ou peu de limitations & & $2,3\left(1,4\right.$ à 3,6) ${ }^{\mathrm{a}}$ & $2,2(1,4 \text { à } 3,6)^{a}$ & $1,6(1,1$ à 3,0$)$ \\
\hline \multicolumn{5}{|l|}{ Type de trouble } \\
\hline Humeur et anxiété & & 1,0 (Réf) & 1,0 (Réf) & 1,0 (Réf) \\
\hline Humeur seulement & & $1,1(0,8$ à 1,6$)$ & $1,2(0,8$ à 1,6$)$ & $1,0(0,7$ à 1,4$)$ \\
\hline Anxiété seulement & & $1,3(0,9$ à 1,9$)$ & $1,3(0,9$ à 1,9$)$ & $1,1(0,8$ à 1,7$)$ \\
\hline \multicolumn{5}{|l|}{ Exercice } \\
\hline $\begin{array}{l}\text { A commencé à faire de l'exercice, } \\
\text { puis a continué tous les jours }\end{array}$ & & & $1,5(0,8$ à 2,6$)$ & \\
\hline $\begin{array}{l}\text { A commencé à faire de } \\
\text { l'exercice, puis a continué } 4 \text { à } 6 \\
\text { fois par semaine }\end{array}$ & & & $1,6(0,9$ à 2,7$)$ & \\
\hline $\begin{array}{l}\text { A commencé à faire de } \\
\text { l'exercice, puis a continué } 2 \text { à } 3 \\
\text { fois par semaine ou moins }\end{array}$ & & & $1,3(0,8$ à 2,1$)$ & \\
\hline $\begin{array}{l}\text { A commencé à faire de } \\
\text { l'exercice, mais a cessé d'en faire }\end{array}$ & & & 1,0 (Réf) & \\
\hline $\begin{array}{l}\text { N'a jamais commencé à faire de } \\
\text { l'exercice }\end{array}$ & & & $1,0(0,6$ à 1,5$)$ & \\
\hline \multicolumn{5}{|c|}{ A adopté de bonnes habitudes de sommeil après avoir reçu le diagnostic } \\
\hline Oui & & & $0,8(0,6$ à 1,1$)$ & \\
\hline Non & & & 1,0 (Réf) & \\
\hline
\end{tabular}

Suite page suivante troubles de l'humeur ou d'anxiété pour éprouver un sentiment de bien-être malgré la maladie.

Offrir une aide aux Canadiens souffrant d'un trouble de l'humeur ou d'anxiété pour leur permettre d'intégrer des réseaux de soutien social solides ou d'en bâtir et les aider à élaborer des stratégies d'adaptation efficaces sont vraisemblablement des objectifs importants à la fois pour les professionnels de la santé mentale et pour les Canadiens atteints de ces troubles. De plus, continuer à miser sur les déterminants sociaux du bien-être, dont le revenu, est tout à fait fondé.

\section{Conflits d'intérêts}

Aucun.

\section{Références}

1. Commission de la santé mentale du Canada. Changer les orientations, changer des vies : stratégie en matière de santé mentale pour le Canada. Calgary (AB) : Commission de la santé mentale du Canada; 2012.

2. Ryan RM, Deci EL. On happiness and human potentials: a review of research on hedonic and eudaimonic wellbeing. Annu Rev Psychol. 2001;52(1): 141-166.

3. Lakaski C, Trottier M. Developing an operational definition of positive mental health: final report based on consultation and workshop [rapport non publié]. Ottawa (Ont.) : Agence de la santé publique du Canada; 2009.

4. Organisation mondiale de la santé (OMS). 10 faits sur la santé mentale [Internet]. Genève : OMS [août 2014]. En ligne à : http://www.who.int /features/factfiles/mental_health/fr/

5. WHOQOL group. The World Health Organization quality of life assessment (WHOQOL): position paper from the World Health Organization. Soc Sci and Med. 1995;41(10):1403-1409.

6. Goldberg JF, Harrow M. Subjective life satisfaction and objective functional outcome in bipolar and unipolar mood disorders: a longitudinal analysis. J Affective Disord. 2005;89(1-3):79-89. 
TABLEAU 3 (suite)

Modèles de régression logistique pour la prédiction de résultats de grande satisfaction à l'égard de la vie (SV) chez les Canadiens de 18 ans et plus atteints d'un trouble anxieux ou de l'humeur

\begin{tabular}{|c|c|c|c|}
\hline & \multicolumn{3}{|c|}{ SV } \\
\hline & $\begin{array}{c}\text { Modèle } 2 \\
\text { RC (IC à } 95 \text { \%) }\end{array}$ & $\begin{array}{c}\text { Modèle } 3 \\
\text { RC (IC à } 95 \%)\end{array}$ & $\begin{array}{c}\text { Modèle } 4 \\
\text { RC (IC à } 95 \% \text { ) }\end{array}$ \\
\hline \multicolumn{4}{|c|}{ Nombre d'heures de sommeil (habituellement) par nuit } \\
\hline Moins de 6 heures & & 1,0 (Réf) & \\
\hline 6 heures à moins de 9 heures & & $1,2(0,8$ à 1,8$)$ & \\
\hline 9 heures ou plus & & $1,5(0,9$ à 2,3$)$ & \\
\hline \multicolumn{4}{|c|}{ Recours à la méditation comme mesure d'autogestion de la maladie } \\
\hline Oui & & $1,0(0,7$ à 1,4$)$ & \\
\hline Non & & 1,0 (Réf) & \\
\hline \multicolumn{4}{|l|}{ Stress perçu (journées) } \\
\hline Pas du tout stressantes & & & $1,8(0,9$ à 3,7$)$ \\
\hline Pas tellement stressantes & & & $2,2(1,5 \text { à } 3,4)^{\mathrm{a}}$ \\
\hline Un peu stressantes & & & $1,5(1,1 \text { à } 2,1)^{a}$ \\
\hline Assez ou extrêmement stressantes & & & 1,0 (Réf) \\
\hline \multicolumn{4}{|l|}{ Adaptation } \\
\hline Grande capacité d'adaptation & & & $1,9(1,4 \text { à } 2,7)^{\mathrm{a}}$ \\
\hline Faible capacité d'adaptation & & & 1,0 (Réf) \\
\hline \multicolumn{4}{|l|}{ Soutien social perçu } \\
\hline Grand soutien social & & & $1,9(1,3 \text { à } 2,8)^{\mathrm{a}}$ \\
\hline Peu de soutien social & & & 1,0 (Réf) \\
\hline
\end{tabular}

Abréviations : IC, intervalle de confiance; Q, quintile; RC, rapport de cotes; Réf, groupe de référence; SV, satisfaction à l'égard de la vie.

Remarques : La satisfaction à l'égard de la vie a été considérée comme élevée si les répondants lui attribuaient un score de 8 ou plus sur une échelle de 0 à 10 .

Le modèle 1 est le modèle de départ englobant seulement les variables sociodémographiques. Le modèle 2 intègre les variables sociodémographiques et les variables de santé et de fonctionnement. Le modèle 3 intègre ces variables ainsi que les comportements d'autogestion. Le modèle 4 intègre les variables sociodémographiques, les variables de santé et de fonctionnement et les variables du stress, de l'adaptation et du soutien social, mais n'inclut pas les variables d'autogestion.

a Statistiquement significatif à $p<0,05$.

7. Sarris J, O'Neil A, Coulson CE, Schweitzer I, Berk M. Lifestyle medicine for depression. BMC Psychiatry. 2014;14:107.

8. Ströhle A. Physical activity, exercise, depression and anxiety disorders. J Neural Transm. 2009;116(6):777-784.

9. Bartholomew JB, Morrison D, Ciccolo JT. Effects of acute exercise on mood and well-being in patients with major depressive disorder. Med Sci Sports Exerc. 2005;37(12):2032-2037.

10. Galper DI, Trivedi MH, Barlow CE, Dunn AL, Kampert JB. Inverse association between physical inactivity and mental health in men and women. Med Sci Sports Exerc. 2006;38(1): 173-178.
14. Hamilton NA, Nelson CA, Stevens N, Kitzman H. Sleep and psychological well-being. Soc Indic Res. 2007;82(1): 147-163.

15. Ryff CD. Psychological well-being in adult life. Curr Dir Psychol Sci. 1995; 4(4):99-104

16. Peth J, Regen F, Bajbouj M, Heuser I, Anghelescu I, Hornung OP. The influence of daytime napping versus controlled activity on the subjective well-being of patients with major depression. Psychiatry Res. 2012; 200(2-3):368-373

17. Leung NT, Lo MM, Lee TM. Potential therapeutic effects of meditation for treating affective dysregulation. Evid Based Complement Alternat Med. 2014;2014:1-7.

18. Galante J, Galante I, Bekkers M, Gallacher J. Effect of kindness-based meditation on health and well-being: a systematic review and meta-analysis. J Consult Clin Psychol. 2014;82(6): 1101-1114.

19. Suto M, Murray G, Hale S, Amari E, Michalak EE. What works for people with bipolar disorder? Tips from the experts. J Affect Disord. 2010;124(1): 76-84.

20. Brown GW, Harris TO (dir.). Life events and illness. New York : Guilford Press; 1989.

21. Brown GW, Andrews B, Harris T, Adler Z, Bridge L. Social support, self-esteem and depression. Psychol Med. 1986; 16(04):813-831. review of the anxiolytic effects of exercise for anxiety and its disorders. Depress Anxiety. 2013;30(4):362-373.

12. Knapen J, Sommerijns E, Vancampfort $\mathrm{D}$, et al. State anxiety and subjective well-being responses to acute bouts of aerobic exercise in patients with depressive and anxiety disorders. Br J Sports Med. 2009;43(10):756-759.

13. American Psychiatric Association. Diagnostic and statistical manual of mental disorders. 5e éd. Washington (DC) : American Psychiatric Publications; 2013.
22. Schiffrin HH, Nelson SK. Stressed and happy? Investigating the relationship between happiness and perceived stress. J Happiness Stud. 2010;11(1): 33-39.

23. Folkman S, Moskowitz JT. Coping: pitfalls and promise. Annu Rev Psychol. 2004;55:745-774.

24. Sin NL, Lyubomirsky S. Enhancing well-being and alleviating depressive symptoms with positive psychology interventions: a practice-friendly metaanalysis. J Clin Psychol. 2009;65(5): 467-487. 
25. Kuehner C, Buerger C. Determinants of subjective quality of life in depressed patients: the role of selfesteem, response styles, and social support. J Affect Disord. 2005;86(2): 205-213.

26. Panayiotou G, Karekla M. Perceived social support helps, but does not buffer the negative impact of anxiety disorders on quality of life and perceived stress. Soc Psychiatry Psychiatr Epidemiol. 2013;48(2):283-294.

27. O’Donnell S, Cheung R, Bennett K, Lagacé C. Enquête sur les personnes ayant une maladie chronique au Canada - Composante des troubles de l'humeur et d'anxiété, 2014 : aperçu de la méthodologie. Promotion de la santé et prévention des maladies chroniques. 2016;36(12):307-21.

28. Statistique Canada. Santé mentale perçue, selon le groupe d'âge et le sexe [Internet]. Ottawa (Ont.) : Statistique Canada; [modifié le 7 mars 2016]. En ligne à : http://www.statcan.gc.ca /tables-tableaux/sum-som/102/cst01 /health110d-fra.htm.

29. Orpana H, Vachon J, Dykxhoorn J, McRae L, Jayaraman G. Surveillance de la santé mentale positive et de ses facteurs déterminants au Canada : élaboration d'un cadre d'indicateurs de surveillance de la santé mentale positive. Promotion de la santé et prévention des maladies chroniques. 2016;36(1):1-10.

30. Diener E. Subjective well-being: the science of happiness and a proposal for a national index. Am Psychol. 2000;55(1):34-43.

31. House JS, Umberson D, Landis KR. Structures and processes of social support. Ann Rev Sociol. 1988;14:293-318.

32. Cutrona CE, Russell DW. The provisions of social support and adaptation to stress. Advances Personal Relation. 1987;1:37-67.

33. Jeste DV, Blazer DG, First M. Agingrelated diagnostic variations: need for diagnostic criteria appropriate for elderly psychiatric patients. Biol Psychiatry. 2005;58(4):265-71.
34. Diener E, Tay L, Oishi S. Rising income and the subjective well-being of nations. J Pers Soc Psychol. 2013; 104(2):267-76.

35. Lindström M, Rosvall M. Marital status, social capital, economic stress, and mental health: a population-based study. Soc Sci J. 2012;49:339-342.

36. Leach LS, Butterworth P, Olesen SC, Mackinnon A. Relationship quality and levels of depression and anxiety in a large population-based survey. Soc Psychiatry Psychiatr Epidemiol. 2013;48(3):417-425. 